\title{
ESTUDIO BIBLIOMÉTRICO DE LA PRODUCCIÓN CIENTÍFICA Y USO DE LA REVISTA CHILENA DE NUTRICIÓN A TRAVÉS DE LA RED SCIELO (2002 A 2007)
}

\author{
BIBLIOMETRIC STUDY OF SCIENTIFIC PRODUCTION \\ AND USE OF REVISTA CHILENA DE NUTRICION \\ TROUGHOUT THE SCIELO NETWORK (2002-2007)
}

Vicente Tomás-Casterá (1), Javier Sanz-Valero (1,2), Carmina Wanden-Berghe $(3,4)$

(1) Departamento de Enfermería Comunitaria

Medicina Preventiva y Salud Pública e Historia de la Ciencia. Universidad de Alicante, Alicante, España.

(2) Departamento de Salud Pública.

Historia de la Ciencia y Ginecología. Universidad Miguel Hernández, Elche, España.

(3) Departamento de Fisiología, Farmacología y Toxicología. Universidad Cardenal Herrera CEU, Elche, España.

(4) Hospital Virgen de los Lirios, Alcoy, España.

\begin{abstract}
Objective: To Identify the main bibliometric indicators from Revista Chilena de Nutrición during the period 2002 to 2007. Methods: Bibliometric analysis about production and use of scientific information published through SciELO network in the mentioned period. Results: There were 213 papers published, of which 105 (49.30\%) were original and the productivity index was 2.02. There were 60 different institutions whose authors have published in the periodical. The predominant language was Spanish, totaling 207 papers $(97,18 \%)$. We counted 328 keywords (PC), from which $328(43,44 \%)$ corresponded to DeCS or MeSH descriptors. There were 5 papers (2.35\%) with all keywords equivalent to descriptors. Analyzing these keywords a clear trend was observed. The obsolescence of references, measured by the median, is 6 years and the Price Index is 48,19\%. Conclusions: Revista Chilena de Nutricion keeps within bibliometric indicators appropriate to health sciences journals. It presents excellent results of the actuality of the bibliographic references and a good rate of original articles.
\end{abstract}

Key words: Revista Chilena de Nutrición, bibliometrics, bibliometric indicators, journal article, access to information, medical subject headings.

Este trabajo fue recibido el 2 de Marzo de 2009 y aceptado para ser publicado el 25 de Mayo de 2010.

\section{INTRODUCCIÓN}

Las revistas científicas desempeñan un importante papel en el proceso de comunicación científica, ya que constituyen la principal vía para la validación del nuevo conocimiento y hacen posible su difusión dentro de la comunidad de investigadores, siendo el principal medio de comunicación, en muchas áreas científicas.

La comunicación científica se realiza, tradicionalmente, a través de la publicación de artículos en revistas especializadas, que constituyen el vehículo por excelencia de la comunicación del conocimiento científico. Por este motivo, el estudio de las publicaciones de un centro, área, comunidad o país, es un indicador válido para cuantificar la actividad científica de dichas unidades $(1,2)$.

La utilidad del análisis de las publicaciones para el estudio de la actividad investigadora y tecnológica ha llevado a la bibliometría a experimentar un gran desarrollo. Estos trabajos permiten estar al tanto y valorar la producción científica de un área de conocimiento y entre sus principales aplicaciones se encuentra el área de la política científica, siendo además utilizados en los procesos de evaluación de los resultados de la actividad investigadora. En todo caso, es necesario que los indica- 
dores de producción, al igual que el resto de los indicadores de Ciencia y Tecnología, sean recopilados con una metodología común, generalmente aceptada, para que sus resultados puedan ser comparados y comparables.

Asimismo, el peritaje de una publicación periódica a través de indicadores bibliométricos permite obtener unos resultados objetivos de gran interés, así como observar las tendencias que sigue la revista. Los datos que obtienen y las conclusiones que se deducen pueden ayudar a los responsables de la revista a mejorar la gestión de la misma, facilitando la toma de decisiones en aspectos relacionados, por ejemplo, con la selección de artículos (3). Aunque, hay que tener presente que en el análisis cuantitativo de datos es posible generar representaciones estadísticas de los fenómenos no plenamente justificadas, puesto que también dependen del juicio y la habilidad del investigador, y de la conveniencia de la pregunta contestada por el dato recogido (4).

Dada la importancia y tradición de los temas de nutrición para los países Iberoamericanos es conveniente acercarse a la realidad de esta actividad científica y de divulgación del conocimiento $(5,6)$, por lo que este trabajo se plantea evaluar, mediante el análisis bibliométrico, la actividad científica, la producción y el consumo de información de la Revista Chilena de Nutrición en el periodo 2002 a 2007.

\section{MATERIAL Y MÉTODO}

Estudio descriptivo transversal de los resultados obtenidos del análisis bibliométrico de los artículos publicados en la Revista Chilena de Nutrición desde enero de 2002 hasta diciembre de 2007, ambos inclusive.

Los datos se obtuvieron mediante la consulta, a través de la Red, de la versión electrónica en la Red SciELO [http://www.scielo.cl/scielo.php?pid=07177518\&script=sci_serial].

La distribución geográfica, al igual que la filiación institucional, se determinó teniendo en cuenta el primer firmante del artículo.

Para estudiar las referencias bibliográficas se procedió al cálculo del tamaño muestral mediante la estimación de parámetros poblacionales en una población infinita $(\mathrm{p}=$ valor esperado $=0,5 ; \mathrm{e}=$ precisión del intervalo 0,05; nivel de confianza $=0,95$ ). Muestra resultante igual a 386 referencias. El método de muestreo fue aleatorio simple sin reemplazo, tomando como base el número total de referencias bibliográficas en la revista. Indicadores estudiados:

- Periodicidad de las publicaciones de la revista.

- Tipología documental publicada.

- Índice de productividad (logaritmo del número de trabajos originales publicados).
- Distribución geográfica de procedencia de los artículos.

- Filiación institucional del primer firmante

- Índice de Lotka o productividad institucional, (clasificación de las instituciones en tres niveles de rendimiento según número de artículos generados: pequeños productores, o Índice de Transitoriedad si presentan un único trabajo; medianos productores, si presentan entre 2 y 9 trabajos y grandes productores, las instituciones con 10 ó más trabajos).

- Número de autores por artículo e índice de colaboración (cociente entre el número de firmas y el número de trabajos).

- Idioma de publicación.

- Palabras clave utilizadas y su relación con los Descriptores de Ciencias de la Salud (DeSC) o su equivalencia con los Medical Subject Headings (Mesh) del Thesaurus de la U.S. National Library of Medicine.

- Estudio de las referencias bibliográficas (RB): tipo de publicación, año de la RB, revistas referidas, idioma de la RB, Índice de firmas, edad de las $\mathrm{RB}$, Semiperiodo de Burton y Kleber (Mediana de la distribución del conjunto de las referencias ordenadas por antigüedad), Índice de Price (porcentaje de referencias con edad igual o menor de 5 años) y núcleo principal de Bradford (conjunto de revistas de mayor pertinencia para un área del conocimiento).

- Análisis de los datos: estudio descriptivo de los indicadores fijados mediante el cálculo de las frecuencias y porcentajes de las variables indicadas, representándose las más relevantes mediante la utilización de tablas y gráficos. Las variables cuantitativas se describieron con su Media y Desviación Standard y las cualitativas con su valor absoluto y porcentaje. Se utilizó la Mediana, como medida de tendencia central, y los Percentiles para determinar el punto de corte que establezca diferentes grupos. Para la introducción y análisis de los datos se usó el programa SPSS versión 15 para Windows. El control de la calidad de la información se efectuó a través de dobles tablas, corrigiendo mediante la consulta con los originales los errores detectados.

\section{RESULTADOS}

La estructura de la Revista Chilena de Nutrición recogida en la Red SciELO consta en 2002, 2003 y 2004 de 3 números, más 1 suplemento (volúmenes 29 a 31); 3 números en el año 2005 (volumen 32); 3 números más 2 suplementos en el año 2006 (volumen 33) y 4 números en el año 2007 (volumen 34). 


\section{Producción científica y tipología documental}

Durante el periodo estudiado se publicaron en Rev Chil Nutr un total de 213 artículos. El año 2006 fue el más productivo con 55 artículos, mientras que el 2002 resultó el de menor productividad con 29, (tabla 1).

El número de originales fue de $105(49,30 \%)$ siendo el índice de productividad de 2,02 y la media de 17,50 originales/año, presentando diferencias entre las distintas anualidades, desde un máximo de $26(47,27 \%)$ en 2006 a un mínimo de $9(31,03 \%)$ en 2002, (gráfico 1).

\section{Procedencia geográfica y filiación institucional}

La distribución geográfica, de los autores que aparecen como primer firmante, es chilena en 152 artículos
$(71,36 \%)$, presentando procedencia no Iberoamericana 2 trabajos $(0,94 \%)$, uno proveniente de Gran Bretaña y otro de USA, (tabla 2). Solo autores de Chile y Argentina han publicado artículos todos los años estudiados.

Se identificaron 60 instituciones con trabajos publicados, presentando una clasificación según el Índice de Lotka con tres niveles de rendimiento: pequeños productores, o Índice de Transitoriedad (un único trabajo), donde encontramos 41 centros $(68,33 \%)$; medianos productores (entre 2 y 9 trabajos) con 16 centros $(26,67 \%)$; y grandes productores (10 ó más trabajos) donde localizamos 3 centros $(5,00 \%)$; en 8 trabajos $(13,33 \%)$ no constaba la institución de filiación, (tabla 3 ).

\section{TABLA 1}

Tipología documental de los artículos publicados en Revista Chilena de Nutrición entre 2002 y 2007.

$\begin{array}{lcccccccc}\text { Tipo de artículo } & \mathbf{2 0 0 2} & \mathbf{2 0 0 3} & \mathbf{2 0 0 4} & \mathbf{2 0 0 5} & \mathbf{2 0 0 6} & \mathbf{2 0 0 7} & \text { Total } & \mathbf{\%} \\ \text { Original } & 9 & 11 & 16 & 18 & 26 & 25 & 105 & 49,296 \\ \text { Revisión } & 13 & 11 & 9 & 10 & 12 & 10 & 65 & 30,516 \\ \text { Opinión } & 2 & 10 & 2 & 2 & 12 & - & 28 & 13,146 \\ \text { Correspondencia } & 5 & - & 2 & 2 & 5 & - & 14 & 6,573 \\ \text { Reseña de libros } & - & - & 1 & - & - & - & 1 & 0,469 \\ \text { Total } & \mathbf{2 9} & \mathbf{3 2} & \mathbf{3 0} & \mathbf{3 2} & \mathbf{5 5} & \mathbf{3 5} & \mathbf{2 1 3} & \mathbf{1 0 0 , 0 0}\end{array}$

\section{GRÁFICO 1}

Trabajos publicados en Revista Chilena de Nutrición de 2002 a 2007: originales y resto de trabajos.

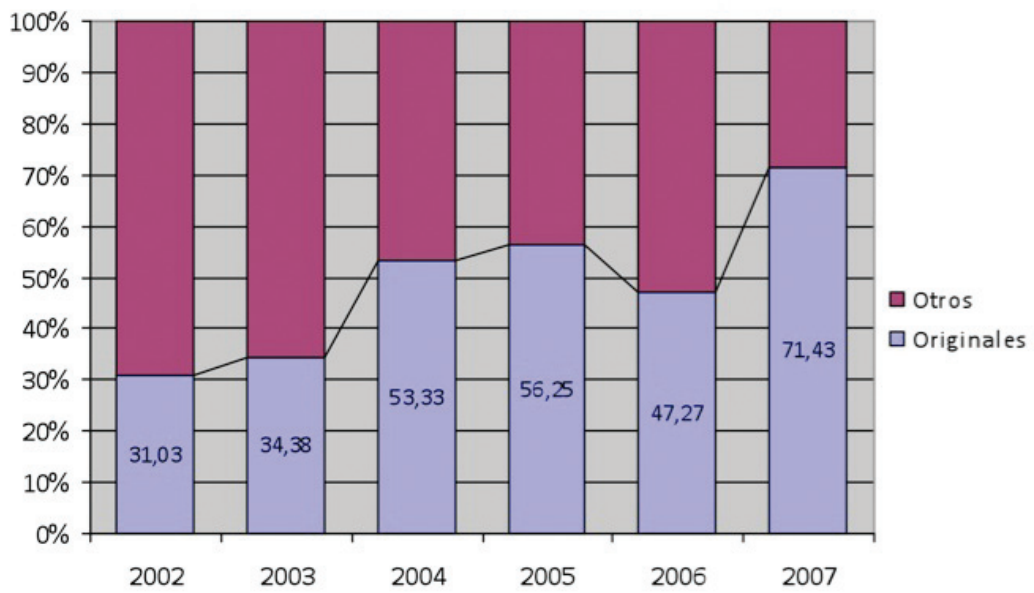


Autoría

Se contabilizaron un total de 613 firmantes, con un Máximo de 12, siendo la Media (equivalente al Índice de cooperación) de 2,88 $\pm 0,13$ (IC95\% 2,63 - 3,13). La Mediana es igual a 3 y la Moda de 1 . El Percentil 25 es de 1 autor y el Percentil 75 de 4 autores.

En los artículos originales se sumó un total de 407 firmantes, con Máximo de 9 y Media de 3,88 \pm 0,17 (IC95\% 3,55 - 4,20). La Mediana y la Moda son ambas de 3 autores. El Percentil 25 y el Percentil 75 son de 3 y 5 autores respectivamente.

\section{Idioma de publicación}

El idioma predominante es el español con 207 artículos $(97,18 \%)$, recogiendo 4 artículos en inglés $(1,88 \%)$ y 2 en portugués $(0,94 \%)$.

\section{Palabras clave}

De los 368 artículos estudiados 34 (15,96\%) no presentaban Palabras Clave (PC), mientras que hubo un

\section{TABLA 2}

Distribución geográfica de los trabajos publicados en Revista Chilena de Nutrición entre 2001 y 2007, según el primer autor.

\begin{tabular}{lcccccccc}
\hline País & $\mathbf{2 0 0 2}$ & $\mathbf{2 0 0 3}$ & $\mathbf{2 0 0 4}$ & $\mathbf{2 0 0 5}$ & $\mathbf{2 0 0 6}$ & $\mathbf{2 0 0 7}$ & Total & $\mathbf{\%}$ \\
\hline Alemania & 1 & - & - & - & - & - & 1 & 0,47 \\
Argentina & 1 & 1 & 2 & 1 & 2 & 3 & 10 & 4,69 \\
Brasil & 1 & 2 & 1 & - & 3 & - & 7 & 3,29 \\
Chile & 26 & 28 & 23 & 23 & 28 & 24 & 152 & 71.36 \\
Colombia & - & - & 1 & - & 8 & 3 & 12 & 5,63 \\
Costa Rica & - & - & - & 1 & 1 & 1 & 3 & 1,41 \\
Cuba & - & - & - & - & 1 & - & 1 & 0,47 \\
España & - & - & - & 1 & 1 & - & 2 & 0,94 \\
Gran Bretaña & - & - & - & - & 1 & - & 1 & 0,47 \\
México & - & - & 1 & 4 & 1 & 2 & 8 & 3,76 \\
Nicaragua & - & - & - & - & - & 1 & 1 & 0,47 \\
Panamá & - & 1 & - & - & - & - & 1 & 0,47 \\
Paraguay & - & - & 1 & - & - & - & 1 & 0,47 \\
Perú & - & - & - & - & 1 & - & 1 & 0,47 \\
USA & - & - & - & - & 1 & - & 1 & 0,47 \\
Venezuela & - & - & - & 1 & 2 & 1 & 4 & 1,88 \\
No consta & - & - & 1 & 1 & 5 & - & 7 & 3,29 \\
Total & $\mathbf{2 9}$ & $\mathbf{3 2}$ & $\mathbf{3 0}$ & $\mathbf{3 2}$ & $\mathbf{5 5}$ & $\mathbf{3 5}$ & $\mathbf{2 1 3}$ & $\mathbf{1 0 0 , 0 0}$
\end{tabular}

\section{TABLA 3}

Instituciones con más de 10 trabajos publicados en Revista Chilena de Nutrición entre 2002 y 2007.

\section{Institución}

Instituto de Nutrición y Tecnología de los Alimentos

Universidad de Chile

Pontificia Universidad Católica de Chile

\section{Total trabajos}

72

33,80

23

10,80

12 
trabajo que presentó 7 PC, correspondiente al máximo.

En total se contabilizaron $755 \mathrm{PC}$ de las que 328 $(43,44 \%)$ se correspondían con términos DeCS o
$\mathrm{MeSH}$, presentando solo 5 artículos $(2,35 \%)$ todas las PC equivalentes a Descriptores. Las dos PC más usadas fueron obesidad y nutrición, (tabla 4). Las PC que fueron

\section{TABLA 4}

Palabras clave utilizadas 5 ó más veces en los artículos publicados en Revista Chilena de Nutrición entre enero 2002 y diciembre 2007, y su equivalencia con los Medical Subject Headings o Descriptores de Ciencias de la Salud.

\begin{tabular}{lcccc}
\hline Palabra clave & $\mathbf{f}_{\mathbf{0}}$ & $\mathbf{\%}$ & MeSH & DeCS \\
Obesidad & 17 & 2,18 & $\mathrm{Si}$ & $\mathrm{Si}$ \\
Nutrición* & 15 & 1,92 & $\mathrm{No}$ & $\mathrm{Si}$ \\
Dieta & 8 & 1,02 & $\mathrm{Si}$ & $\mathrm{Si}$ \\
Niños** & 8 & 1,02 & $\mathrm{No}$ & $\mathrm{No}$ \\
Frutas & 7 & 0,89 & $\mathrm{Si}$ & $\mathrm{Si}$ \\
Chile & 6 & 0,77 & $\mathrm{Si}$ & $\mathrm{Si}$ \\
Hábitos alimentarios & 6 & 0,77 & $\mathrm{No}$ & $\mathrm{No}$ \\
Promoción & 6 & 0,77 & $\mathrm{No}$ & $\mathrm{Si}$ \\
Promoción de la Salud & 16 & 0,77 & $\mathrm{Si}$ & $\mathrm{Si}$ \\
Actividad física & 5 & 0,64 & $\mathrm{No}$ & $\mathrm{Si}$ \\
Alimentos funcionales & 5 & 0,64 & $\mathrm{Si}$ & $\mathrm{Si}$ \\
Colombia & 5 & 0,64 & $\mathrm{No}$ & $\mathrm{Si}$ \\
Embarazo & 5 & 0,64 & & \\
\hline * No es un MeSH o DeSC pero es un sinónimo aceptado & & & \\
** El Descriptor es niños (en singular) & & & &
\end{tabular}

TABLA 5

Distribución porcentual de la tipología documental referida en Revista Chilena de Nutrición entre 2002 y 2007.

\section{Tipo documental}

Artículo original

Memoria e informe

Capítulo de libro

Libro

Página web

Tesis

En prensa

Monografía en Internet

Programa informático

\section{$\%$}

73,58

10,88

5,70

5,18

1,81

1,04

0,78

0,52

0,52
IC $95 \%$

$69,18-77,97$

$7,77-13,99$

$3,39-8,01$

$2,97-7,39$

$0,48-3,14$

$0,03-2,05$

$0,00-1,65$

$0,00-1,23$

$0,00-1,23$ 
utilizadas en primer lugar en un mayor número de oportunidades fueron frutas y nutrición: 4 ocasiones $(1,88 \%)$.

\section{Referencias bibliográficas}

El número total de referencias listadas en los documentos publicados fue de 5197, estando escritas 185 de ellas $(3,56 \%)$ en formato electrónico, lo que representa una relación de 28:1. Presenta Mínimo de 0 y Máximo de 85 referencias. La Media de referencias por artículo es de 24,40 \pm 1,20 (IC95\% 22,03 - 26,77). La Mediana igual a 22 y la Moda es de 0 referencias. El Percentil 25 y el Percentil 75 son de 13 y 32 referencias respectivamente.

La muestra estudiada (386 referencias) mostró que la tipología documental más citada fueron los artículos de revista, 73,58\% de los sucesos (IC95\% 69,18 - 77,97), (tabla 5).

El número de autores por referencia presenta una Mediana de 3 y Moda de 1, advirtiéndose que 13,73\% (IC95\% 10,30 - 17,16) de las referencias presentan más de 6 firmantes.

El idioma de las referencias es el inglés en $64,77 \%$ (IC95\% 60,00 - 69,53), seguido del español 34,72\% (IC95\% 29,97 - 39,46) y alemán con $0,26 \%$ (IC95\% 0,00- 0,77).

La obsolescencia de las referencias citadas en Revista Chilena de Nutrición, medida por la Mediana
(Semiperiodo de Burton y Kleber), es de 6 años (gráfico 2), y el Índice de Price es del 48,19\%.

El estudio de la dispersión de la literatura científica citada, y por tanto referida, determina la concentración de un porcentaje aproximado de documentos en un número diferente de revistas: núcleo principal, 14 revistas $(7,18 \%)$ que recogen 106 referencias $(33,23 \%)$, (tabla 6$)$. El núcleo 2 con 52 revistas $(26,67 \%)$ recoge 84 referencias $(26,33 \%)$ y el núcleo 3 con 129 revistas $(66,15 \%)$ contiene 129 referencias $(40,44 \%)$, (gráfico 3). El porcentaje de autocitas es de 5,99\% (IC95\% 3,23 - 8,74).

En el estudio de consumo se observó que el 16,15\% (IC95\% 11,96 - 20,55) de las referencias tenían mal redactada la abreviatura, internacionalmente aceptada, de la revista referenciada.

\section{DISCUSIÓN}

Es primordial señalar la disponibilidad, de los datos estudiados, del texto completo de la producción científica publicada en formato electrónico, en la Red SciELO.

Revista Chilena de Nutrición es una publicación que como muestran los datos de producción, presenta un ligero crecimiento en los últimos años. El enfoque iberoamericano de esta revista es incuestionable. Pero, el escaso número de artículos con filiación externa al

\section{GRÁFICO 2}

\section{Edad de las referencias bibliográficas contenidas en Revista Chilena de Nutrición según año de publicacióny resto de trabajos.}

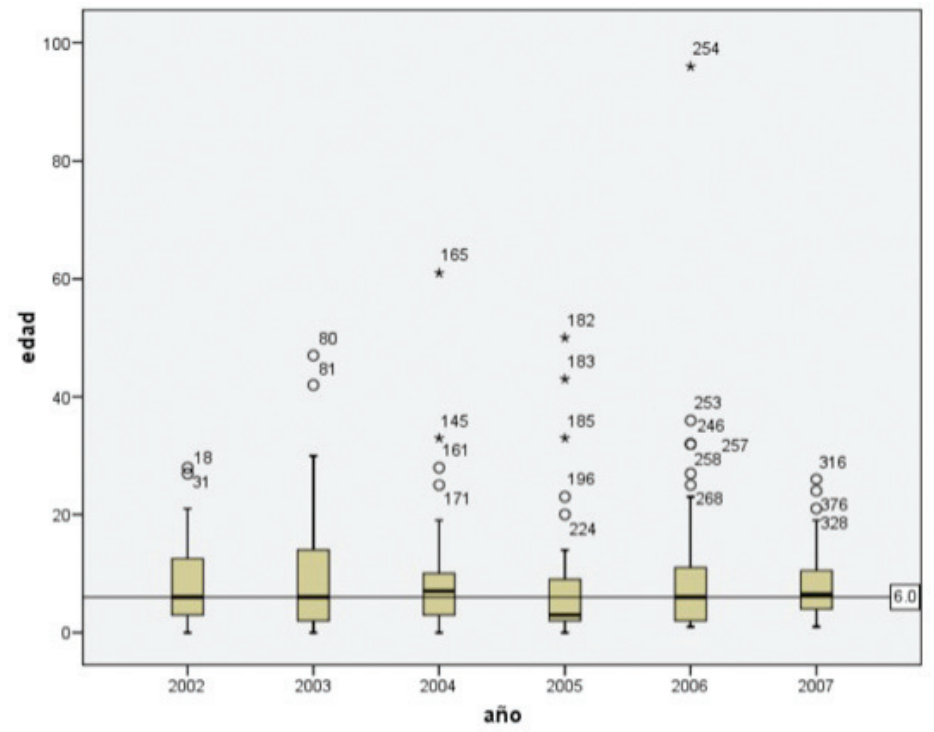




\section{TABLA 6}

Revistas referenciadas en Revista Chilena de Nutrición entre 2002 y 2007, pertenecientes al núcleo principal de Bradford.

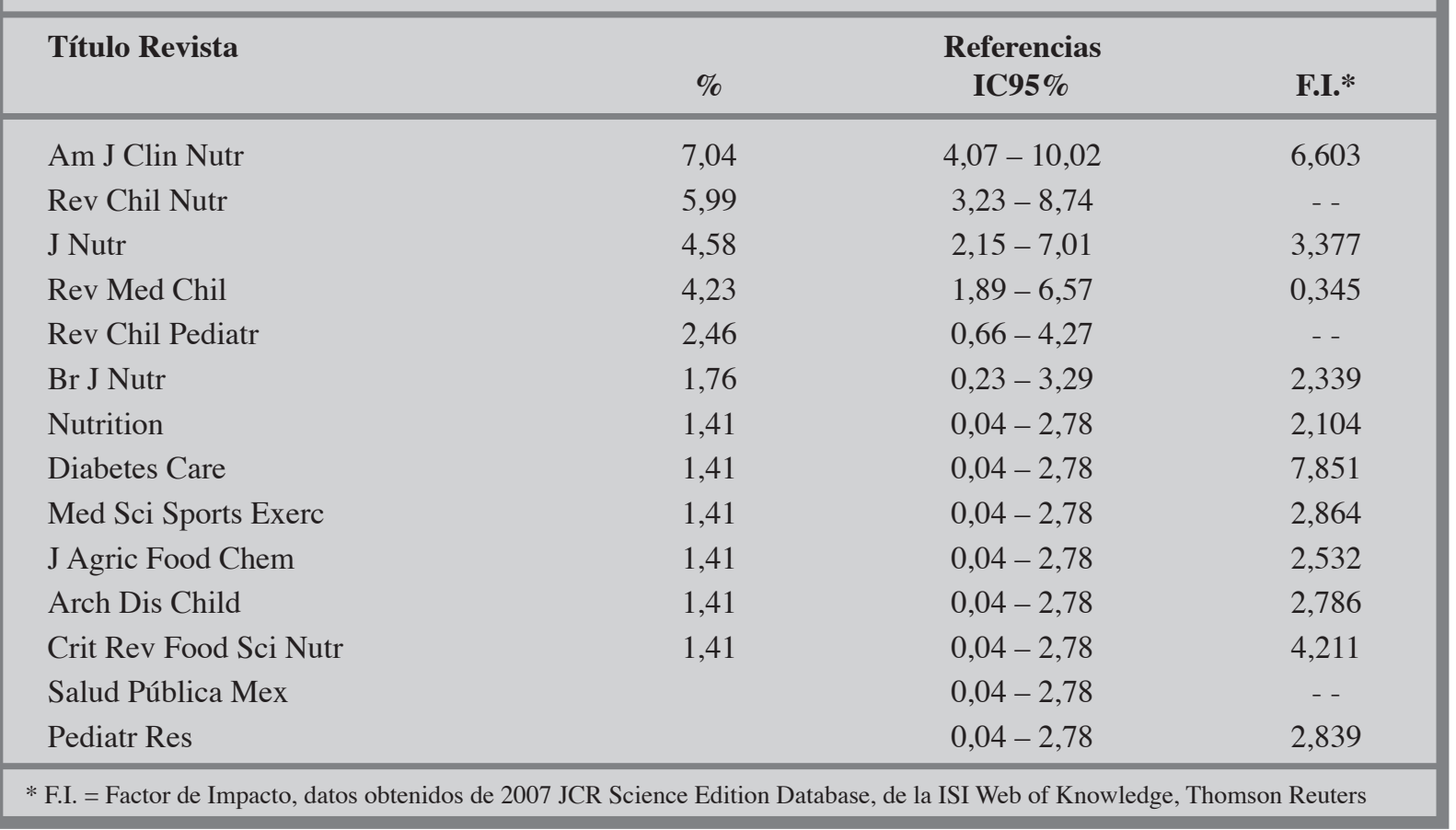

\section{GRÁFICO 3}

Dispersión de las revistas y los artículos referidos, en los anillos de Bradford, en Revista Chilena de Nutrición en los años 2002 a 2007según año de publicacióny resto de trabajos.

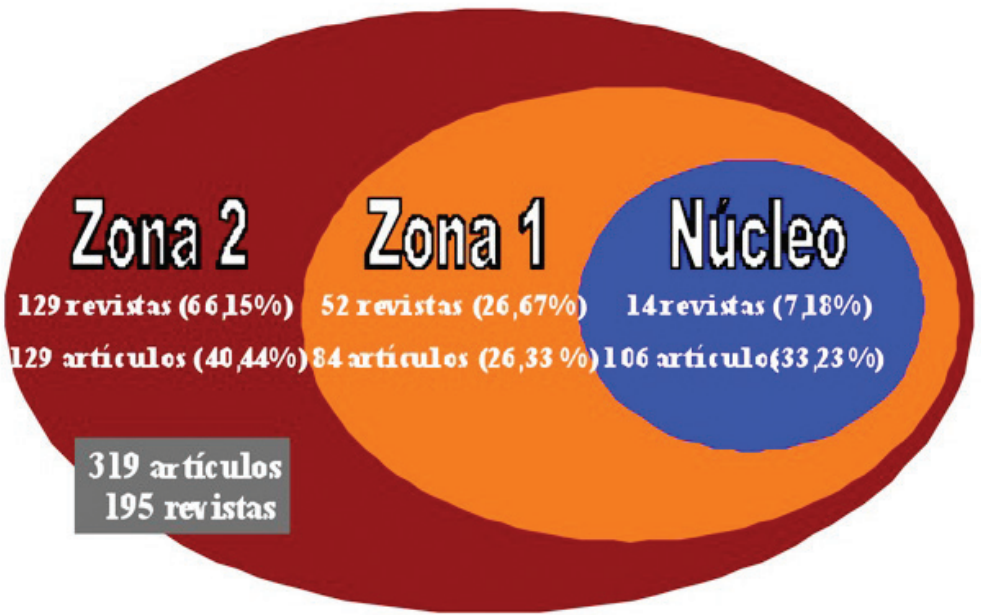


ámbito chileno debe ser debatido por el equipo editorial de la revista. La orientación de la revista es claramente comunitaria, basta con ver las principales Palabras Clave utilizadas para comprobarlo.

La producción científica calculada, el número de autores y el índice de cooperación presentan datos similares a otra revistas sobre ciencias de la salud del área iberoamericana $(3,7)$. Bien es cierto, que la proporción de artículos originales supera levemente los valores habituales $(1,8)$.

El predominio del español es una constante en las revistas iberoamericanas sobre nutrición. Aquellos autores con una capacidad idiomática mayor, tienden a publicar en revistas de habla anglófona una vez realizado el esfuerzo de escribir el artículo en inglés. Además, pueden ser amparados por su propia institución que impulsa y recomienda el envío a revistas de alto impacto aunque ello suponga tener que pagar por la posterior recuperación del artículo o incluso por su revisión (9).

Los resultados sobre la falta de relación entre las Palabras Clave y Descriptores de Ciencias de la Salud o con Medical Subject Headings son también similares a lo observado hasta el momento en revistas iberoamericanas sobre nutrición e incluso del campo de las ciencias de la salud $(10,11)$. Este dato denota un posible desconocimiento de los Descriptores por parte de los autores que debería ser vigilado por el equipo editorial, con el fin de promover su correcta utilización y por tanto mejorar la indización de los artículos en las bases de datos bibliográficas.

Las tasas anuales de referencias por publicación son acordes a resultados de estudios previos (12-14), teniendo en cuenta siempre las limitaciones impuestas por las normas para los autores. También es normal el mayor uso de citar artículos de revistas científicas para respaldar los trabajos, hecho acrecentado por las bases de datos bibliográficas (MEDLINE, LILACS, etc).

Las revistas más referidas coinciden con publicaciones sobre las ciencias de la nutrición. Asimismo, el hecho de que se refieran artículos publicados en revistas de alto impacto ya ha sido estudiado (15), es por tanto esperable que este grupo de revistas concentren el mayor de citas y que en consecuencia se sitúen en el núcleo principal de Bradford, reafirmando la importancia que los autores relacionados con el campo de la nutrición dan a las publicaciones periódicas de mayor consulta.

Revista Chilena de Nutrición muestra porcentajes de autocitas por debajo de los estudios bibliométricos ya citados en este artículo. Si bien, es lógico que los autores que publican en una revista la citen, ya que divulga temas relacionados con su área de conocimiento (16). En todo caso, siempre hay que tener pensar que mejorar la visibilidad científica de los autores, complacer a superiores, a editores o revisores e incluir citas de prestigio con la intención de incrementar el peso de las evidencias son otros motivos que influyen en la selección de la bibliográfica $(17,18)$. Nada nuevo que no se sepa y se haya comprobado $(19,20)$.

El análisis de la obsolescencia, medido tanto por la Mediana como por el Índice de Price, muestra buenos resultados, lo que denota una actualidad de las referencias utilizadas.

En conclusión, Revista Chilena de Nutrición, presenta unos indicadores bibliométricos similares a los de las revistas punteras del ámbito iberoamericano y totalmente equiparable a las publicaciones del ámbito de las ciencias de la salud. Hay que destacar los excelentes resultados de actualidad de las referencias bibliográficas. Al tiempo, presenta un buen porcentaje de artículos originales en relación a la tipología documental.

\section{RESUMEN}

Objetivo: Conocer los principales indicadores bibliométricos de la Revista Chilena de Nutrición en el periodo 2002 a 2007. Método: Análisis bibliométrico de la actividad, la producción de información científica y de consumo de los documentos publicados e indizados en la Red SciELO, en el periodo de estudio. Resultados: Se publicaron un total de 213 artículos. El número de originales fue de $105(49,30 \%)$ siendo el índice de productividad de 2,02. Se identificaron 60 instituciones con trabajos publicados. El idioma predominante es el español con 207 artículos $(97,18 \%)$. Se contabilizaron 755 Palabras Clave (PC) de las que $328(43,44 \%)$ se correspondían con términos DeCS o MeSH, presentando solo 5 artículos $(2,35 \%)$ todas las PC equivalentes a Descriptores. Las dos PC más usadas fueron obesidad y nutrición. El estudio de las PC muestra una orientación claramente comunitaria. La obsolescencia de las referencias citadas, medida por la Mediana, es de 6 años y el Índice de Price es del 48,19\%. Conclusiones: Revista Chilena de Nutrición, presenta unos indicadores bibliométricos totalmente equiparables a las publicaciones del ámbito de las ciencias de la salud. Hay que destacar los excelentes resultados de actualidad de las referencias bibliográficas. Al tiempo, presenta un buen porcentaje de artículos originales en relación a la tipología documental.

Palabras clave: Bibliometría, indicadores bibliométricos, artículo de revista, acceso a la información, descriptores en ciencias de la salud.

Dirigir la correspondencia a:

Señor

Javier Sanz-Valero. 
Departamento de Enfermería Comunitaria,

Medicina Preventiva y Salud Pública e

Historia de la Ciencia.

Universidad de Alicante.

Campus de Sant Vicent del Raspeig.

Ap. Correos 99 - E 03080.

Alicante (España).

E-mail: javier.sanz@ua.es

\section{BIBLIOGRAFÍA}

1. Pérez Andrés C, Estrada Lorenzo JM, Villar Álvarez F, Rebollo Rodríguez MJ. Estudio bibliométrico de los artículos originales de la Revista Española de Salud Pública (1991-2000), parte primera: indicadores generales. Rev Esp Salud Pública 2002;76(6):659-72.

2. Osca Lluch J. Estudio bibliométrico de las revistas electrónicas españolas de las áreas de ciencias sociales y humanidades y desarrollo de un sistema informático para la elaboración de índices de citas de revistas españolas de forma automática [monografía en Internet]. Valencia: Dirección General de Universidades, Ministerio de Educación y Ciencia [Proyecto EA2005- 0126]; 2005. Disponible en: http://univ.micinn.fecyt.es/univ/proyectos2005/ EA2005-0126.pdf

3. Granda-Orive JI, García Río F, Gutiérrez Jiménez T, Jiménez Ruiz CA, Solano Reina S, Sáez Valls R. Análisis y evolución de los indicadores bibliométricos de producción y consumo del área de tabaquismo a través de Archivos de Bronconeumología (período 1970-2000): Comparación con otras áreas neumológicas. Arch Bronconeumol 2002;38(11):523-9.

4. Romo M, Castillo C. Metodologías de las ciencias sociales aplicadas al estudio de la nutrición. Rev Chil Nutr 2002;29(1):14-22.

5. Consejo Editorial Iberoamericano. El idioma español en la ciencia. Nutr Hosp 2006;21(1):1.

6. Muzzo S. Situación actual y planes futuros de las revistas de nutrición en Iberoamerica. Rev Chil Nutr 2008;35(1):8-9.

7. de Lorenzo-Cáceres A, Otero Puime A. Publicaciones sobre evaluación de la atención primaria en España tras veinte años de reforma (1984-2004): análisis temático y bibliométrico. Rev Esp Salud Pública. 2007;81(2):131-45.

8. Valera Garrido JF, de la Gala Sánchez F. Análisis bibliométrico de la productividad en la revista Mapfre Medicina. Mafre Med 2001;12(3):157-67.

9. González de Dios J. Evaluación de la calidad de la evidencia científica en Anales Españoles de Pediatría. An Esp Pediatr 2001;54(6):605.

10. Tomás Casterá V, Sanz-Valero J, Juan-Quilis V, Wanden-Berghe C, Culebras JM, García de Lorenzo A; Grupo de Comunicación y Documentación Científica en Nurición CDC-Nut SENPE. Estudio bibliométrico de la revista Nutrición Hospitalaria en el periodo 2001 a 2005: Parte I, análisis de la producción científica. Nutr Hosp 2008;23(5):469-76.

11. Granda-Orive JI, García Río F, Callol Sánchez L. Importancia de las Palabras Clave en las búsquedas bibliográficas. Rev Esp Salud Publica 2003;77(6):765-7.

12. Granda-Orive JI, García Río F, Roig Vázquez F, Escobar Sacristán J, Gutierrez Jiménez T, Callol Sánchez L. Las Palabras Clave como herramientas imprescindibles en las búsquedas bibliográficas: Análisis de las áreas del sistema respiratorio a través de Archivos de Bronconeumología. Arch Bronconeumol 2005;41(2);78-83.

13. Miralles J, Ramos JM, Ballester R, Belinchón I, Sevilla A, Moragón M. Estudio bibliométrico de la revista Actas Dermo-Sifiliográficas (1984-2003). Análisis de las referencias bibliográficas. Actas Dermosifiliogr 2005;96(9):563-71.

14. Villar Álvarez F, Estrada Lorenzo JM, Pérez Andrés C, Rebollo Rodríguez MJ. Estudio bibliométrico de los artículos originales de la Revista Española de Salud Pública (1991-2000): Parte tercera: análisis de las referencias bibliográficas. Rev Esp Salud Publica 2007;81(3):247-59.

15. Tomás Casterá V, Sanz-Valero J, Juan-Quilis V, Wanden-Berghe C, Culebras JM, García de Lorenzo A; Grupo de Comunicación y Documentación Científica en Nurición CDC-Nut SENPE. Estudio bibliométrico de la revista Nutrición Hospitalaria en el periodo 2001 a 2005: Parte II, análisis de consumo; las referencias bibliográficas. Nutr Hosp 2008;23(6):541-6.

16. Callaham M, Wears RL, Weber E. Journal prestige, publication bias, and other characteristics associated with citation of published studies in peer-reviewed journals. JAMA. 2002;287(1):2847-50.

17. Iglesias Vázquez E, Culebras JM, García de Lorenzo A. Evaluación de Nutrición Hospitalaria (III): tiempos de demora, materias y citación. Nutr Hosp 2002;17(1):34-42.

18. Castiel LD, Sanz-Valero J. Entre fetichismo e sobrevivência: o artigo cientifico é uma mercadoria acadêmica?. Cad Saúde Pública 2007;23(12):304150.

19. Aleixandre Benavent R, Valderrama Zurián JC, 
Miguel-Dasit A, Granda Orive JI. El factor de impacto de Revista Iberoamericana de Micología. Rev Iberoam Micol 2004;21:161-7.

20. Kiserud T, Nylenna M. Citations and reference lists: for whom and for what purpose? Ultrasound Obstet
Gynecol 2003;22(2):105-7.

21. Culebras Fernández JM, García de Lorenzo A, Wanden-Berghe C, Castiel LD, Sanz-Valero J. ¡Cuidado!, sus referencias bibliográficas pueden ser estudiadas. Nutr Hosp 2008;23(2):85-8. 